\title{
A relação entre vida acadêmica e a motivação para aprender em universitários
}

\author{
Acácia Aparecida Angeli dos Santos \\ Jocemara Ferreira Mognon \\ Thatiana Helena de Lima \\ Neide Brito Cunha
}

\begin{abstract}
Resumo
O presente estudo propôs identificar as relações entre a vida acadêmica e a motivação para aprendizagem e possíveis diferenças em razão do sexo, idade e curso dos estudantes. Participaram 239 universitários que foram avaliados com a Escala de Avaliação da Vida Acadêmica (EAVA) e a Escala de Motivação para Aprendizagem (EMAPRE). Os resultados indicaram correlação significativa e positiva entre a meta aprender e o fator habilidade do estudante, e negativa para os dois fatores: envolvimento em atividades não obrigatórias; condições para o estudo e desempenho acadêmico. Para a meta performance-aproximação, houve correlação significativa e positiva com o fator condições para o estudo e desempenho acadêmico. Na meta performance-evitação, houve correlações positivas e significativas com dois fatores: compromisso com o curso; condições para o estudo e desempenho acadêmico. Foram encontradas diferenças significativas em relação ao sexo e ao curso. Os resultados foram discutidos à luz da literatura.
\end{abstract}

Palavras-chave: Motivação, estudantes universitários, rendimento escolar.

\section{The relationship between academic life and motivation for learning in college}

\begin{abstract}
In this study we aim at identifying the relationship between academic life and motivation for learning by evaluating possible differences based on student's sex, age and course. We assessed 239 students using the "Escala de Avaliação da Vida Acadêmica (EAVA) and the "Escala de Motivação para Aprendizagem" (EMAPRE). The results indicated that the correlation between instruments were significant, being positive between learning goal and the student's ability and negative for two factors: involvement in non-compulsory activities and conditions in academic performance. There was significant and positive correlation for the performance-approach goal between conditions for studying and academic achievement. Regarding performance-avoidance goal, there were significant positive correlations between the factors: commitment to the course, conditions for studying and academic performance. There were significant differences regarding student's sex and course. Based on literature in the area, we discussed the results of the research.
\end{abstract}

Key words: Motivation, college students, academic achievement.

\section{La relación entre vida académica y la motivación para aprender en universitarios}

\section{Resumen}

Este estudio se propuso identificar las relaciones entre la vida académica y la motivación para el aprendizaje y las posibles diferencias en razón del sexo, edad y carrera de los estudiantes. Participaron 239 universitarios que fueron evaluados con la Escala de Evaluación de Vida Académica (EAVA) y con la Escala de Motivación para Aprendizaje (EMAPRE). Los resultados indicaron correlación significativa y positiva entre la meta aprender y el factor habilidad del estudiante, y negativa para los dos factores: envolvimiento en actividades no obligatorias; condiciones para el estudio y rendimiento académico. Para la meta performance-aproximación, hubo correlación significativa y positiva con el factor condiciones para el estudio y rendimiento académico. En la meta performance-evitación, hubo correlaciones positivas y significativas con dos factores: compromiso con la carrera; condiciones para el estudio y rendimiento académico. Se encontraron diferencias significativas en relación al sexo y a la carrera. Los resultados se discutieron de acurdo con la literatura.

Palabras clave: Motivación, estudiantes universitarios, rendimiento escolar. 


\section{Introdução}

A realização de um curso superior tem sido cada vez mais valorizada no mercado de trabalho. No entanto, Franco (2008) constatou, com base em dados do Instituto Nacional de Estudos e Pesquisas Educacionais (INEP), no ano de 2007, que a média de evasão do ensino superior brasileiro se concentrou em $18,5 \%$. Esse processo é recorrente tanto em instituições públicas $(12,4 \%)$ como em particulares (26\%). Dessa forma, a evasão é considerada um problema e a busca de suas causas tem sido objeto de muitas pesquisas educacionais (Silva Filho, Motejunas, Hipólito, \& Lobo, 2007).

Diante do exposto, torna-se importante contextualizar o ambiente de aprendizagem e compreender como as experiências universitárias marcam a vida acadêmica. A preocupação com a vida acadêmica se dá em decorrência da necessidade de efetiva aprendizagem, a qual vai depender de variáveis de grande influência social. Santos, Polydoro, Teixeira e Bardagi (2010) apontam ser difícil avaliar os efeitos da educação superior, devido ao fato de que a identificação do impacto da universidade é dependente do perfil do estudante e de suas características e, por outro lado, da diversidade das instituições, cursos e experiências de formação superior. Assim sendo, esse construto tem sido entendido ora como um fator psicológico, ou um conjunto de fatores, ora como um processo (Zenorini \& Santos, 2010a).

Para alguns autores (Almeida \& cols., 2000; Cunha \& Carrilho, 2005), as dificuldades encontradas no contexto universitário são de diversas ordens, dentre elas estão as questões pessoais dos alunos, as exigências acadêmicas que devem ser desempenhadas com eficiência e a adaptação a um novo ambiente, fatores estes que influenciam tanto o desempenho acadêmico como o desenvolvimento psicossocial dos estudantes. Isso pode prejudicar a motivação para aprender dos alunos e o professor desempenha um papel importante nesse processo.

Como se pode perceber, a integração à vida acadêmica é um processo multifacetado, construído nas relações estabelecidas entre o estudante e a instituição de ensino. Este processo é vivenciado e percebido de forma particular (Polydoro, 2000) e envolve expectativas em relação à estrutura, normas, qualidade no ensino e o prestígio da universidade diante da comunidade (Polydoro, Primi, Serpa, Zaroni, \& Pombal, 2001). Para Tinto (1975), a interação entre o estudante e o sistema acadêmico da instituição ocorre ao longo da graduação, modificando continuamente o compromisso do estudante com a instituição e com sua formação.

A universidade é um espaço de aprendizagem e, segundo Guimarães e Boruchovitch (2004), também de socialização. Assim, o ambiente universitário favorece o desenvolvimento cognitivo e afetivo. Esses e outros fatores, como o estilo do professor em relação ao desenvolvimento de atividades de aprendizagem, à organização curricular e ao contexto acadêmico no geral, são essenciais para a motivação dos alunos. Assim sendo, as ações do professor são elementos informativos que definem o comportamento, o envolvimento, as estratégias de pensamento e o grau de esforço esperado pelos alunos (Almeida \& Soares, 2003).

Em obra recente, Bzuneck (2010) aponta que é preciso mostrar ao aluno o significado e a importância de se estudar tais disciplinas ou conteúdos, assim o professor precisa captar quais são os interesses dos alunos e o que lhes causa curiosidade para que as atividades sejam propostas com características de desafios. Percebe-se, com isso, que o professor precisa estar motivado para conseguir motivar os seus alunos.

Durante toda a graduação, é requisitada aos estudantes uma maior autonomia para participação nas atividades curriculares e extracurriculares, como palestras, cursos, congressos e outros. Nesse momento, são importantes as atribuições motivacionais para realizar com sucesso as atividades. De acordo com Bzuneck (2004), para que o aluno esteja motivado, é preciso que, entre outras opções, ele tenha escolhido o curso que frequenta, estando assim envolvido de forma ativa nas tarefas que levam ao processo de aprendizagem. Esse envolvimento se resume no emprego de esforço no processo de aprender, persistindo a cada tarefa. Em contrapartida, o autor complementa que o universitário que deixa de investir esforço mínimo exigido pela atividade desiste quando a tarefa é percebida como mais exigente.

Procurando compreender as motivações dos estudantes, surgiu, na década de 70 do século $X X$, a Teoria de Metas de Realização, que busca explicações para a qualidade do envolvimento do aluno no processo de aprendizagem. Conceitualmente, busca entender o propósito que leva o estudante a se envolver em uma tarefa, sendo relevantes as diferenças individuais (Zenorini \& Santos, 2004). Assim, a meta de realização que a pessoa adota representa o motivo ou a razão pela qual ela realizará determinada tarefa (Bzuneck, 2004).

Para Accorsi, Bzuneck e Guimarães (2007), as metas de realização surgem em decorrência da percepção que os estudantes têm do seu contexto de aprendizagem. Os autores complementam que o contexto percebido pode estar relacionado ao estilo do professor, identificado por suas ações, verbalizações e formas de avaliação, e também ao contexto universitário, como as normas estabelecidas pela universidade, a disponibilidade de eventos e o incentivo para atividades extracurriculares. Dessa forma, Archer (1994) já ressaltava que o contexto escolar pode levar à ocorrência das duas metas, por exemplo, quando o assunto for de menor interesse para o estudante, ele se utilizará da meta performance para atingir o desempenho esperado e, em situações em que sentir maior interesse em se aprofundar no assunto, aplicará a meta aprender.

A meta aprender caracteriza-se nos alunos que direcionam mais energia as suas atividades, valorizam o conhecimento, buscam melhorar suas habilidades (Accorsi \& cols., 2007) e interpretam o erro como um alerta para adotar novas estratégias de aprendizagem. Nesse sentido, estudos têm mostrado que o estudante que se utiliza dessa meta costuma recorrer a estratégias de aprendizagem mais adequadas para atingir os seus objetivos (Bzuneck, 2005; Zenorini \& Santos, 
2004). No entanto, há estudantes mais preocupados com os aspectos externos, característica da meta performance, a qual pode ser divida em duas, em razão de suas especificidades. O aluno caracterizado pela meta performance-aproximação é apontado como aquele que sente necessidade de se destacar entre os colegas mostrando-se mais inteligente, sem que o seu foco primordial esteja na aprendizagem efetiva. Já os orientados pela meta performance-evitação objetivam salvaguardar-se da desvalorização por parte de professores e colegas, preocupam-se primordialmente em não parecerem incompetentes e apresentam comportamentos menos adaptativos (Bzuneck, 2004).

Os altos índices de desistências nos cursos superiores evidenciam a necessidade de compreensão das variáveis presentes na vida acadêmica dos estudantes. Como a pesquisa de Oliveira, Souza, Vieira, Adário e Rezende (2007), com 655 estudantes ingressantes de vários cursos, com o objetivo de identificar as variáveis tanto de contexto como motivacionais nos universitários por meio de questões fechadas sobre renda familiar, custeio, expectativa e motivação para o curso. Os resultados mostraram que é importante compreender como o estudante interpreta e vivencia a fase de permanência na universidade, com seus desafios acadêmicos, sociais, pessoais e vocacionais.

O desenvolvimento do estudante além da sala de aula, como a participação em palestras, cursos, congressos, projetos de extensão, de iniciação científica e outros, também tem sido foco de algumas pesquisas como a de Almeida e cols. (2000), que realizaram um estudo com 223 universitários para investigar como o envolvimento em atividades extracurriculares influenciava na vida acadêmica, na adaptação ao curso e no desempenho acadêmico. Os resultados indicaram que os alunos que participavam das atividades extracurriculares apresentaram maior disciplina com os estudos, melhor relacionamento com colegas, autonomia e adaptação à universidade. Fior e Mercuri (2004) também buscaram investigar o envolvimento em atividades extracurriculares e as mudanças pessoais percebidas em 16 universitários. Os resultados demonstraram que as experiências educacionais ultrapassam os limites da sala de aula e das exigências curriculares obrigatórias e que as experiências extracurriculares contribuíram de forma diferenciada para as mudanças no universitário, categorizadas em cinco domínios: conhecimentos e habilidades acadêmicas, complexidade cognitiva, competência prática, competência interpessoal e humanitarismo. A partir dos resultados, as autoras ressaltam a importância da universidade na concretização das propostas de formação que integrem também atividades extracurriculares.

Na revisão de literatura realizada por Terezini, Pascarela e Blimling (1996), nos Estados Unidos, sobre a influência das atividades extracurriculares na aprendizagem e no desenvolvimento cognitivo do estudante, alguns resultados revelaram benefícios desse tipo de atividade no desenvolvimento pessoal do aluno. Mas verificaram que é necessária uma estruturação dessas atividades para que não atrapaIhem a aprendizagem, como o excesso de trabalho, de trei- nos esportivos e de contato com os pares que apresentam uma ação negativa no desenvolvimento acadêmico e intelectual dos alunos.

O estudo de Cunha e Carrilho (2005), utilizando-se do QVA (Questionário de Vivência Acadêmica), teve como objetivo compreender as relações entre a vivência acadêmica e o rendimento estudantil. Foi realizado com 100 alunos do curso de Engenharia Militar e revelou que o rendimento acadêmico pode ser influenciado pela qualidade das vivências dos estudantes em nível pessoal e de realização acadêmica, estando menos relacionadas ao contexto universitário. No entanto, Teixeira, Dias, Wottrich e Oliveira (2008), investigando por meio de entrevistas individuais a experiência de adaptação à universidade em calouros, ressaltam que o contexto universitário tem um papel importante a desempenhar no processo de adaptação à universidade. Dessa forma, é fundamental oferecer informações relativas à vida acadêmica para que o aluno possa usufruir dos benefícios oferecidos pela universidade.

Com o objetivo de investigar melhor o sucesso acadêmico universitário, Harackiewicz, Barron, Tauer e Eliot (2002) fizeram um estudo longitudinal com 471 alunos e investigaram as metas de realização, medidas de inteligência, interesse e desempenho acadêmico dos alunos do primeiro ao último ano de graduação. Na primeira etapa, foram identificadas as variáveis preditivas do interesse e do desempenho dos alunos em uma disciplina introdutória de Psicologia, oferecida no primeiro semestre do curso de graduação. Depois houve um acompanhamento dos alunos até que eles se formassem para examinar o interesse contínuo em Psicologia. Tanto as metas de realização quanto as medidas de inteligência e o desempenho prévio no Ensino Médio contribuíram com os resultados iniciais e ao longo do curso. A meta aprender associou-se ao interesse contínuo, enquanto que a meta performance-aproximação previu o desempenho acadêmico.

Descrever as vivências acadêmicas em alunos de Psicologia também foi o objetivo da pesquisa de Igue, Bariani e Milanesi (2008). Participaram 203 estudantes do $1^{\circ}$ e $5^{\circ}$ ano e o instrumento utilizado foi o Questionário de Vivência Acadêmica - versão reduzida (QVA-r). Encontrou-se, nos resultados, que os estudantes com expectativas mais altas apresentaram avaliação melhor das vivências acadêmicas por estarem mais dispostos e motivados para investirem seus recursos nas atividades. Houve médias baixas para os dois grupos no fator institucional, no qual os alunos declararam não conhecer os serviços oferecidos pela universidade. Dessa forma, os autores sugerem que medidas devam ser tomadas para que não ocorram problemas como desinteresse, desmotivação e má adaptação.

Visando ampliar a compreensão de variáveis que compõem o contexto universitário, o objetivo deste estudo foi investigar a correlação entre os construtos, vida acadêmica e motivação para aprender. Ao lado disso, pretendeu, ainda, levantar possíveis diferenças na vida acadêmica e na motivação para aprendizagem, em razão das variáveis sexo, idade e curso frequentado pelos universitários. 


\section{Método}

\section{Participantes}

A escolha da amostra ocorreu por conveniência. Participaram 239 universitários dos cursos de: Letras $(n=22)$, Arquitetura $(n=30)$, Engenharia Mecânica $(n=30)$, Fisioterapia $(n=44)$, Educação Física $(n=35)$, Pedagogia $(n=23)$, Administração $(n=30)$ e Tecnologia da Informação $(n=25)$. Em relação à variável sexo, 44,4\% $(n=106)$ eram homens e $55,6 \%(n=133)$, mulheres. As idades variaram de 18 a 52 anos ( $M=23,35 ; D P=5,69)$. Os alunos frequentavam os seguintes semestres: $57,3 \%(n=137)$ eram do $2^{\circ} ; 10,5 \%$ $(n=25)$, do $3^{\circ}$ e $32,2 \%(n=77)$, do $4^{\circ}$.

\section{Instrumentos}

Escala de Avaliação da Vida Acadêmica (EAVA) elaborada por Vendramini e cols. (2004). Tem como objetivo captar a autopercepção dos estudantes sobre a sua vivência universitária por meio de itens que envolvem questões contextuais, interacionais e pessoais. É composta de 34 itens, com cinco fatores autoexplicativos. Os itens da escala foram dispostos em uma escala Likert de cinco pontos, sendo: discordo totalmente (1 ponto), discordo na maioria das vezes (2 pontos), discordo e concordo em igual proporção (3 pontos), concordo na maioria das vezes (4 pontos) e concordo totalmente (5 pontos). A pontuação mínima possível é de 34 pontos e a máxima, de 170 pontos. A análise psicométrica demonstrou resultados satisfatórios, tendo sido encontrado um índice de consistência interna geral de alpha $=0,87$. Os coeficientes de Cronbach por fator são: ambiente universitário (alpha $=0,72$ ); compromisso com o curso (alpha $=0,78$ ); habilidade do estudante (alpha $=0,80$ ); envolvimento em atividades não obrigatórias (alpha $=0,71$ ); e condições para o estudo e desempenho acadêmico (alpha $=0,73$ ).
Escala de Motivação para Aprendizagem (EMAPRE) - elaborada por Zenorini e Santos (2010b). Possui 28 itens constituídos por três tipos de metas, classificadas como: meta aprender, meta performace-aproximação e a meta performace-evitação. As questões do instrumento foram montadas numa escala Likert, envolvendo três opções de resposta, "concordo" (3 pontos), "não sei" (2 pontos) e "discordo" (1 ponto). A pontuação mínima possível é de 28 pontos e a máxima, de 84 pontos. A análise fatorial exploratória identificou itens com carga fatorial acima de 0,45. A escala atingiu índices de consistência interna aceitáveis, meta aprender (alpha=0,80), meta performance-aproximação (alpha $=0,76$ ) e meta performance-evitação (alpha $=0,74)$.

\section{Procedimento}

Após a aprovação pelo Comitê de Ética de uma universidade do interior do Estado de São Paulo, foi solicitada a autorização dos coordenadores de cada curso pesquisado para a realização da coleta de dados. Antes da aplicação, foram explicados os objetivos da pesquisa aos estudantes, o caráter voluntário da participação, bem como o sigilo de suas respostas e identidades. Após a assinatura do Termo de Consentimento Livre e Esclarecido (TCLE), os instrumentos foram aplicados de forma coletiva em sala de aula, levando em média 30 minutos.

\section{Resultados}

Os dados foram organizados em uma planilha e submetidos à análise da estatística descritiva e inferencial, conforme os objetivos deste estudo. Para se analisar as relações entre os constructos, foi aplicada a correlação de Pearson. Os resultados são apresentados na Tabela 1.

Tabela 1. Índice de correlação de Pearson ( $r$ ) e nível de significância $(p)$ entre a vivência acadêmica e a motivação para aprendizagem.

\begin{tabular}{|c|c|c|c|}
\hline Fatores & Meta Aprender & $\begin{array}{l}\text { Meta Performance- } \\
\text { aproximação }\end{array}$ & $\begin{array}{l}\text { Meta Performance- } \\
\text { evitação }\end{array}$ \\
\hline Ambiente universitário & 0,102 & 0,070 & 0,090 \\
\hline Compromisso com o curso & 0,055 & 0,036 & $0,127\left(^{*}\right)$ \\
\hline Habilidade do estudante & $0,216\left(^{* *}\right)$ & 0,070 & $-0,108$ \\
\hline $\begin{array}{l}\text { Envolvimento em atividades } \\
\text { não obrigatórias }\end{array}$ & $-0,176\left(^{* *}\right)$ & $-0,009$ & 0,067 \\
\hline $\begin{array}{l}\text { Condições para o estudo e } \\
\text { desempenho acadêmico }\end{array}$ & $-0,210\left(^{* *}\right)$ & $0,192\left(^{* \star}\right)$ & $0,248\left({ }^{* *}\right)$ \\
\hline
\end{tabular}


Tabela 2. Valores de média, desvio-padrão, $t$ e $p$ obtidos com o teste $t$ de Student com os fatores da EAVA e os da EMAPRE, considerando a variável sexo dos estudantes.

\begin{tabular}{|c|c|c|c|c|c|c|}
\hline Fatores & Sexo & $N$ & $M$ & $D P$ & $t$ & $p$ \\
\hline \multirow{2}{*}{ Ambiente universitário } & masculino & 106 & 24.02 & 3.027 & \multirow{2}{*}{$-0,95$} & \multirow{2}{*}{0,341} \\
\hline & feminino & 133 & 24.40 & 3.075 & & \\
\hline \multirow{2}{*}{ Compromisso com o curso } & masculino & 106 & 19.08 & 2.209 & \multirow{2}{*}{$-2,45$} & \multirow{2}{*}{0,015} \\
\hline & feminino & 133 & 19.81 & 2.323 & & \\
\hline \multirow{2}{*}{ Habilidade do estudante } & masculino & 106 & 28.18 & 5.609 & \multirow{2}{*}{0,71} & \multirow{2}{*}{0,475} \\
\hline & feminino & 133 & 27.71 & 4.429 & & \\
\hline \multirow{2}{*}{ Envolvimento em atividades não obrigatórias } & masculino & 106 & 15.51 & 2.126 & \multirow{2}{*}{$-1,57$} & \multirow{2}{*}{0,116} \\
\hline & feminino & 133 & 15.95 & 2.137 & & \\
\hline \multirow{2}{*}{$\begin{array}{l}\text { Condições para o estudo e desempenho } \\
\text { acadêmico }\end{array}$} & masculino & 106 & 11.27 & 3.091 & \multirow{2}{*}{$-3,12$} & \multirow{2}{*}{$<0.001$} \\
\hline & feminino & 133 & 12.53 & 3.079 & & \\
\hline \multirow{2}{*}{ Total da vida acadêmica } & masculino & 106 & 98.07 & 8.510 & \multirow{2}{*}{$-2,30$} & \multirow{2}{*}{0,022} \\
\hline & feminino & 133 & 100.4 & 7.133 & & \\
\hline \multirow{2}{*}{ Meta Aprender } & masculino & 106 & 15.61 & 3.482 & \multirow{2}{*}{$-0,92$} & \multirow{2}{*}{0,357} \\
\hline & feminino & 133 & 16.03 & 3.462 & & \\
\hline \multirow{2}{*}{ Meta Performance-aproximação } & masculino & 106 & 20.78 & 4.635 & \multirow{2}{*}{$-2,36$} & \multirow{2}{*}{0,019} \\
\hline & feminino & 133 & 22.14 & 4.241 & & \\
\hline \multirow{2}{*}{ Meta Performance-evitação } & masculino & 106 & 18.72 & 3.134 & \multirow{2}{*}{$-1,06$} & \multirow{2}{*}{0,288} \\
\hline & feminino & 133 & 19.11 & 2.502 & & \\
\hline \multirow{2}{*}{ Total da Motivação para Aprendizagem } & masculino & 106 & 55.11 & 7.480 & \multirow{2}{*}{$-2,49$} & \multirow{2}{*}{0,013} \\
\hline & feminino & 133 & 57.28 & 5.961 & & \\
\hline
\end{tabular}

Os dados da Tabela 1 revelaram, em relação à EAVA, que o fator compromisso com o curso apresentou correlação estatisticamente significativa com a meta performance-evitação, enquanto que o fator habilidade do estudante apresentou correlações positivas e significativas com a meta aprender. Já o fator envolvimento em atividades não obrigatórias apresentou correlações negativas e significativas com a meta aprender. Para o último fator, condições para o estudo e desempenho acadêmico, foram encontradas correlações negativas e significativas com a meta aprender e positivas e significativas com as metas performance-aproximação e evitação.

Procurou-se, também, analisar possíveis diferenças entre a amostra estudada. Para saber se havia diferença entre os estudantes com relação à variável sexo, aplicou-se a prova t-test de Student, cujos resultados estão apresentados na Tabela 2.

É possível observar, na Tabela 2, que os homens obtiveram médias superiores em relação às mulheres apenas no fator habilidade do estudante. Verificou-se que as mu-
Iheres obtiveram diferenças estatisticamente significativas nos fatores compromisso com o curso $(p<0,015)$, condições para o estudo e desempenho acadêmico $(p<0,002)$ e no total da $\operatorname{EAVA}(p<0,022)$. A mesma análise foi feita nas metas da EMAPRE, e os resultados novamente indicaram diferenças significativas para as mulheres na meta performance-aproximação $(p<0,019)$ e no total da escala $(p<0,013)$.

Recorreu-se à análise de variância (ANOVA) para se aferir possível diferenças na pontuação dos instrumentos, considerando o curso dos estudantes. No que se refere à vida acadêmica, nos fatores ambiente universitário e envolvimento em atividades não obrigatórias, foram constatadas diferenças estatisticamente significativas entre os diferentes cursos, sendo elas, respectivamente, $[F(7,231)=21,307$; $p=0,023]$ e $[F(7,231)=13,304 ; p=0,004]$. O teste de post-hoc de Tukey indicou que, no fator ambiente universitário, os alunos do curso de Arquitetura obtiveram médias maiores que os do curso de Pedagogia e, no fator envolvimento em atividades não obrigatórias, o curso de Administração obteve médias superiores que o curso de Fisioterapia. 
Quanto à escala de motivação para aprendizagem, houve diferença estatisticamente significativa nas metas performance-aproximação e performance-evitação, respectivamente, $[F(7,231)=41,696 ; p=0,038]$ e $[F(7,231)=17,079$; $p=0,031]$. Pelo teste post-hoc de Tukey, observou-se que as diferenças estavam nas médias que foram superiores para os alunos do curso de Pedagogia quando comparados aos do curso de Tecnologia da Informação em ambas as metas da EMAPRE.

As idades foram agrupadas em faixas etárias, para tanto foram estabelecidos quatro grupos. O primeiro foi composto por estudantes de 18 a 19 anos (27,2\%) agrupando aqueles que terminaram o ensino médio com uma idade padrão esperada - denominados de tradicionais. O segundo grupo foi composto por estudantes de 20 a 22 anos $(31,4 \%)$ que, por motivos diversos, ingressaram na universidade mais tarde. No terceiro grupo estavam os estudantes de 23 a 26 anos $(19,9 \%)$ e o último grupo foi composto por estudantes de 27 anos ou mais (20,9\%), denominados maduros. Recorreu-se à ANOVA na tentativa de identificar eventuais diferenças, porém não foi encontrada nenhuma que fosse estatisticamente significativa.

\section{Discussão}

Ao mesmo tempo em que é crescente o número de universitários, é também o de evasão da universidade, a qual pode ser ocasionada pela falta de motivação para aprender ou a outras variáveis relacionadas. A motivação tem sido considerada importante porque muitos estudiosos têm apontado os seus benefícios para a aprendizagem (Bzuneck, 2004; 2005; Guimarães \& Boruchovitch, 2004; Zenorini \& Santos, 2004; Zenorini, Santos \& Monteiro (no prelo)). Bzuneck (2004) ressalta que a meta aprender aparece relacionada a resultados positivos, ao esforço e à persistência na busca de conhecimentos e no aprimoramento das habilidades, sendo, desta forma, propulsora de crescimento intelectual. Isso corrobora com os resultados encontrados na presente pesquisa, visto que a meta aprender apareceu relacionada positivamente com a habilidade do estudante, que está ligada à percepção pessoal de competências cognitivas, como capacidade de expressão, compreensão, resolução de problemas e concentração (Vendramini \& cols, 2004). Porém, relacionou-se negativamente com o fator envolvimento em atividades não obrigatórias e com o fator condições para o estudo e desempenho acadêmico.

A respeito das atividades não obrigatórias, muitos têm sido os benefícios apontados, como maior satisfação com o curso, desenvolvimento cognitivo, social e afetivo, além de segurança, autoestima e valores altruísticos, ganho nas habilidades verbais e no domínio de conteúdos específicos (Almeida \& cols, 2000; Fior \& Mercuri, 2004). No entanto, a pesquisa de Terenzini, Pascarella e Blimling (1996) destacou também os malefícios para a aprendizagem do estudante, quando essas atividades não estão ligadas diretamente ao curso frequentado, já que podem ocupar o tempo que seria importante para os estudos. Bzuneck (2004) ressalta que as atividades extracurriculares contribuem para uma meIhor integração, persistência e esforço, mas com tendência ao processamento de superfície, e o estudo de Zenorini e Santos (2004) encontrou a meta aprender correlacionada positivamente com o processamento de estratégias de profundidade (as cognitivas - simples e complexas e metacognitivas) que exigem maior envolvimento do estudante com a aprendizagem. Talvez isso justifique o resultado encontrado na presente pesquisa.

Os resultados encontrados para a meta performance-aproximação são condizentes com a literatura, que se caracteriza pelo estudante buscar demonstrar capacidade, e tiveram relação positiva com o fator condições para o estudo e desempenho acadêmico, ou seja, os estudantes investem o seu tempo na busca por estratégias que acabam influenciando o seu rendimento acadêmico. Nesse sentido, justifica-se a meta performance-aproximação favorecer o desempenho acadêmico em termos de notas. Esse resultado assemelha-se ao encontrado na pesquisa de Harackiewicz e cols. (2002).

Surpreendentemente, a meta performance-evitação apareceu correlacionada positivamente com o fator compromisso com o curso e com o fator condições para o estudo e desempenho acadêmico. Isso pode levar a questionamentos de parecer não haver distinção entre as metas performance-aproximação e performance-evitação, ou de que há a influência de uma sobre a outra. Para Bzuneck (2004), o aluno mede as chances de, com o enfrentamento de desafios, ter comprovações de que é inteligente. Porém, diante do fracasso, este é atribuído à falta de capacidade, e aparecem sentimentos negativos como raiva e vergonha. $O$ autor afirma que ambas as metas se correlacionam de modo independente, no entanto é preciso prudência com relação às afirmações das consequências comportamentais negativas ligadas à meta performance, pois muitas pesquisas têm mostrado resultados contraditórios.

Na presente pesquisa, foram encontradas diferenças estatisticamente significativas nas variáveis sexo e curso. A literatura tem mostrado que cada estudante vivencia de forma diferenciada a sua passagem pela universidade (Polydoro, 2000). Para Almeida e Soares (2003), as diferenciações em relação às motivações, expectativas e níveis de autonomia podem aparecer em grupos de alunos segundo diversas variáveis, como o sexo, o curso e o semestre.

Os dados obtidos mostraram que as mulheres têm maior compromisso com o curso, condições para o estudo e desempenho acadêmico. Isso pode significar uma maior identidade com o curso e autoconhecimento dos seus objetivos, interesses e habilidades pessoais, o que promove maior investimento nas atividades de aprendizagem e, conseqüentemente, melhor rendimento acadêmico. Para Cunha e Carrilho (2005), o rendimento acadêmico pode ser influenciado pela qualidade das vivências. Mas, em contrapartida, verificou-se, na presente pesquisa, que a motivação das estudantes está mais direcionada à possibilidade de mostrar suas capacidades e competências (meta performance-apro- 
ximação). Esse resultado difere dos achados nos estudos de Zenorini e Santos (2010b) e Zenorini e cols. (no prelo), que encontraram mulheres com maior tendência à meta aprender e com atitudes mais positivas em sala de aula e os homens com maior disposição às metas performance- aproximação e evitação.

\section{Considerações finais}

Cada vez mais a universidade tem sido relacionada ao primeiro passo para o sucesso profissional. Os acontecimentos da universidade irão refletir sobre a profissão escolhida e sobre a constituição pessoal dos estudantes, visto que o espaço universitário proporciona, além da aquisição de novos conhecimentos, uma nova visão global da adequação à profissão, o contato com novos valores e crenças, questionamentos e experiências acadêmicas e sociais que proporcionam aos estudantes, principalmente aos mais jovens, amadurecimento pessoal.

Algumas limitações podem ser apontadas neste estudo. Em virtude dos múltiplos fatores associados à vida universitária, é de se esperar que as variáveis envolvidas encontrem-se, em maior ou menor grau, relacionadas entre si, possivelmente se influenciando mutuamente. Nesse sentido, cabe uma ressalva para o fato de a EAVA ter cinco dimensões abrangentes da vida universitária. Provavelmente ela não consegue abranger todas as experiências acadêmicas que podem, por sua vez, relacionar-se com a motivação. Dessa forma, os resultados indicaram correlações de magnitude baixa. Pode-se hipotetizar que, apesar de haver associações entre os fatores da EAVA e as metas da EMAPRE, as baixas correlações sugerem que outros fatores podem explicar melhor a vida acadêmica. Enfim, novas pesquisas são necessárias, que investiguem outras variáveis que podem estar relacionadas à vida acadêmica para que se possa ampliar a compreensão dos fenômenos que ocorrem na universidade e contribuir para a criação de medidas que melhorem a vida acadêmica dos estudantes e diminuam a evasão no nível supe

\section{Referências}

Accorsi, D. M. P., Bzuneck, J. A., \& Guimarães, S. E. R. (2007). Envolvimento cognitivo de universitários em relação à motivação contextualizada. Psico-USF, 12(2), 291-300.

Almeida, L. S., Soares, A. P. C., Vasconcelos, R. M., Capela, J. V., Vasconcelos, J. B., Corais, J. M., \& Fernandes, A. (2000). Envolvimento extracurricular e ajustamento acadêmico: um estudo sobre as vivências dos universitários com e sem funções associativas. Em A. P. Soares, A. Osório, J. V. Capela, L. S. Almeida, R. M. Vasconcelos \& S. M. Caíres (Orgs.), Actas do seminário transição para o ensino superior (pp.167-187). Braga, Portugal.
Almeida, L. S., \& Soares, A. P. (2003). Os estudantes universitários: sucesso escolar e desenvolvimento psicossocial. Em E. Mercuri \& S. A. J. Polydoro (Orgs.), Estudante universitário: características e experiências de formação (pp.15-40). Taubaté, SP: Cabral.

Archer, J. (1994). Achievement goals as a measure of motivation in university students. Contemporary Educational Psychology, 19(4), 430-446.

Bzuneck, J. A. (2004). A motivação do aluno orientado a metas de realização. Em E. Boruchovitch \& J. A. Bzuneck (Orgs.), A motivação do aluno: contribuições da psicologia contemporânea (pp.58-77). Petrópolis, RJ: Vozes.

Bzuneck, J. A. (2005). A motivação dos alunos em cursos superiores. Em M. C. R. A. Joly, A. A. A. dos Santos \& F. F. Sisto (Orgs.), Questões do cotidiano universitário (pp. 217-237). São Paulo: Casa do Psicólogo.

Bzuneck, J. A. (2010). Como motivar os alunos: sugestões práticas. Em E. Boruchovitch, J. A. Bzuneck \& S. E. R. Guimarães (Orgs.). Motivação para aprender. Aplicações no contexto educativo (pp.13-24). Rio de Janeiro: Vozes.

Cunha, S. M., \& Carrilho, D. M. (2005). O processo de adaptação ao ensino superior e o rendimento acadêmico. Psicologia Escolar e Educacional 9(2), 215-224.

Franco, A. P. (2008). Ensino superior no Brasil: cenário, avanços e contradições. Jornal de políticas educacionais, 4, 53-63.

Fior, C. A., \& Mercuri, E. (2004). Formação universitária: o impacto das atividades não-obrigatórias. Em E. Mercuri \& S. A. J. Polydoro, Estudante universitário: características e experiências de formação (pp. 129-154). São Paulo: Cabral.

Guimarães, S. É. R., \& Boruchovitch, E. (2004). O estilo motivacional do professor e a motivação intrínseca dos estudantes: uma perspectiva da Teoria da Autodeterminação. Psicologia, reflexão e crítica, 17(2), 143-150.

Harackewicz, J. M., Barron, K. E., Tauer, J. M., \& Elliot, A. J. (2002). Predicting success in college: a longitudinal study of achievement goals and ability measures as predictors of interest and performance from freshman year through graduation. Journal of Educational Psychology, 94(3), 562-575.

Igue, E. A., Bariani, I. C. D., \& Milanesi, P. V. B. (2008). Vivências acadêmicas e expectativas de universitários ingressantes e concluintes. Psico-USF, 13(2), 155-164.

Instituto Nacional de Estudos e Pesquisas Educacionais Anísio Teixeira - INEP. (2007). Censo da Educação Superior. Recuperado: 01 mar 2008. Disponível: http://www.inep.gov.br/ superior/censosuperior 
Oliveira, S. M. S. S., Souza, O. M. K., Vieira, V. W. V., Adário, Y. S., \& Rezende, M. A. F. S. (2007). Identificação de variáveis de contexto universitário de primeiro ano. PSIC - Revista de Psicologia da Vetor Editora, 8(2). 227-235.

Polydoro, S. A. J. (2000). O trancamento de matrícula na trajetória acadêmica do universitário: condições de saída e de retorno à universidade. Tese de doutorado, Faculdade de Educação, Universidade Estadual de Campinas, Campinas, São Paulo.

Polydoro, S. A. J., Primi, R., Serpa, M. N. F., Zaroni, M. M. H., \& Pombal, K. C. P. (2001). Desenvolvimento de uma escala de integração ao ensino superior. Psico-USF, 6(1), 11-17.

Santos, A. A. A., Polydoro, S. A. J., Teixeira, M. A., \& Bardagi, M. P. (2010). Avaliação da integração do aluno ao ensino superior brasileiro. Em A. A. A. Santos, F. F. Sisto, E. Boruchovitch \& E. Nascimento (Orgs.), Perspectivas em avaliação psicológica (pp.165-188). São Paulo: Casa do Psicólogo.

Silva Filho, R. L. L., Motejunas, P. R., Hipólito, O., \& Lobo, M. B. C. M. (2007). A evasão no ensino superior brasileiro. Cadernos de Pesquisa, 37(132), 641-659.

Teixeira, M. A. P., Dias, A. C. G., Wottrich, S. H., \& Oliveira, A. M. (2008). Adaptação à universidade em jovens calouros. Psicologia Escolar e Educacional, 12(1), 185-202.

Terezini, P. T., Pascarella, E. T., \& Blimling, G. S. (1996). Student's outof-class experiences and their influence on learning and cognitive development: a Literature Review. Journal of College Student Development, 37(2), 149-162.

Tinto, V. (1975). Dropout from higher education: a theorical synthesis of recent research. Review of Educational Research, 45(1), 89125.

Vendramini, C. M. M., Santos, A. A. A., Polydoro, S. A. J., Sbardelini, E. T. B., Serpa, M. N. F., \& Natário, E. G. (2004). Construção e validação de uma escala sobre avaliação da vida acadêmica. Estudos de Psicologia, 9(2), 259-268.

Zenorini, R. P. C., \& Santos, A. A. A. (2004). A motivação e a utilização de estratégias de aprendizagem em universitários. Em E. Mercuri \& S. A. J. Polydoro. Estudante universitário: características e experiências de formação (pp. 67-86). Taubaté, SP: Cabral.

Zenorini, R. P. C., \& Santos, A. A. A. (2010a). Teoria de Metas de Realização: fundamentos e avaliação. Em E. Boruchovitch, J. A. Bzuneck \& S. É. R.Guimarães (Orgs.), Motivação para aprender. Aplicações no contexto educativo (pp. 99-125). Rio de Janeiro: Vozes.

Zenorini, R. P. C., \& Santos, A. A. A. (2010b). Escala de metas de realização da motivação para aprendizagem. Interamerican Journal of Psychology, 44, 291-298.

Zenorini, R. P. C., Santos, A. A. A., \& Monteiro, R. M. (no prelo) Motivação para aprender: relação com o desempenho de estudantes. Paideia.

Recebido em: 18/08/2010

Reformulado em: 13/05/2011

Aprovado em: 08/07/2011

\section{Sobre as autoras}

Acácia Aparecida Angeli dos Santos (acacia.santos@saofrancisco.edu.br / acacia.angeli@gmail.com)

Psicóloga. Doutora em Psicologia Escolar e Desenvolvimento Humano pela USP e Docente da Graduação e do Programa de Pós-Gradução Stricto Sensu em Psicologia da Universidade São Francisco/Itatiba.

\section{Jocemara Ferreira Mognon}

Discente do $5^{\circ}$ ano do curso de Psicologia da Universidade São Francisco e bolsista de Iniciação Científica PIBIC/CNPq

\section{Thatiana Helena de Lima}

Psicóloga e Mestranda de Psicologia da Universidade São Francisco. Bolsista CAPES

\section{Neide Brito Cunha}

Doutora em Psicologia pela Universidade São Francisco

\section{Endereço para correspondência:}

Dra. Acácia A. Angeli dos Santos - Universidade São Francisco - Programa de Pós-Graduação em Psicologia - R. Alexandre Rodrigues Barbosa, 45 - 13251-040 Itatiba - SP. 